\title{
Effect of measuring distance error on working phase voltage at zero potential live working point
}

\author{
Zhu Liang ${ }^{1,2}$, Wang Zhenyu ${ }^{1,2^{*}}$,Yang Qi ${ }^{1,2}$, Guo Hao ${ }^{1,2}, \mathrm{Niu} \mathrm{Jie}^{1,2}$ \\ ${ }^{1}$ Hunan transmission Maintenance Company,Changsha 410100, China \\ ${ }^{2}$ Live Inspection and Intelligent Operation Technology State Grid Corporation Laboratory, Changsha 410100, China.
}

\begin{abstract}
When the live working is carried out in the distribution network, the zero-potential charging operation is often used. The safety of zero-potential live working is closely related to the measurement distance error. The influence of the measurement error of the zero-potential live working point on the operating phase voltage needs to be studied. Based on the Floyd algorithm, a method for calculating the shortest electrical distance from the operating point to the substation is proposed. The sub-station is centered on the branch nodes of the line to form the distance matrix, and the Floyd algorithm is used to calculate the shortest electrical distance from the working point to the substation. The working phase injection voltage is further calculated based on the zero phase potential characteristics of the operating phase. Finally, based on the PSCAD, the system model is established. The simulation concludes that the distance between the substation and the working point is less than $20 \mathrm{~m}$, and the operating point voltage can still be below the safe voltage.
\end{abstract}

\section{Introduction}

With the rapid development of China's power grid technology and the continuous improvement of power supply reliability requirements, the compatible live working technology has made new developments and breakthroughs ${ }^{[1]}$. For the live working of the distribution network, the operating point voltage needs to be kept below the safe voltage. However, due to the existence of the measurement distance error at the zero-potential live working point, the safety of the live working operator is seriously jeopardized ${ }^{[2-3]}$. Therefore, the research on measuring the distance error of the zero potential live working point has received more and more attention and attention.

In order to study the influence of the measurement distance error on the working phase voltage of the zeropotential live working point, in recent years, the research on the measuring method of the live working point has been rapidly developed. In the current study, there are mainly three methods for measuring the distance of live working points: (1) Impedance method. The impedance of the loop is calculated from the voltage and current measured at the operating point in [4]. Since the length of the line is proportional to the impedance, the distance from the working point to the exit of the transformer can be determined. The premise is to ignore the distributed capacitance and leakage conductance of the line. The advantage of the impedance method is that it is relatively simple and reliable, but most impedance methods have accuracy problems ${ }^{[5]}$. (2) Work point analysis method. The distance between the working points is obtained by analysis and calculation using the power frequency voltage and current amount recorded during the charging operation in [6]. According to the required measurement information, the operating point analysis method can be divided into single-ended electric quantity method and double-ended electric quantity method. The single-ended electric quantity method calculates the working point according to the voltage and current of one end and the necessary system parameters. Distance ${ }^{[7]}$, the doubleended electrical quantity method is based on the voltage and current at both ends of the line and the necessary system parameters, and the distance measurement equation is obtained by simplification, and the working point distance is solved ${ }^{[8]}$. (3) Traveling wave method. According to the traveling wave transmission theory, the working point ranging is realized in [9]. The reliability and accuracy of the traveling wave method are theoretically independent of the line type, the grounding resistance of the working point and the systems on both sides, but are subject to many engineering factors in practice. The existing ranging method does not consider the influence of the measurement distance error on the operating phase voltage, which will seriously endanger the safety of the live working operator.

In view of this, a method for calculating the shortest electrical distance from the operating point to the substation is proposed in this paper. Based on the Floyd algorithm, calculate the shortest electrical distance from the operating point to the substation. The working phase injection voltage is further calculated based on the zero phase potential characteristics of the operating phase. Finally, based on the PSCAD simulation software, the 
system model is established. The simulation shows the influence of the measurement error of the zero-potential live working point on the operating phase voltage through the operating phase voltage curve.

\section{Calculation method for shortest electrical distance from live working point of distribution network to substation}

In the above method, it is necessary to know the voltage drop from the substation to the operating point $\Delta \dot{U}$, and it is related to the line unit impedance $\mathrm{z}$ and the electrical distance $l$ from the substation to the working point. The unit impedance of the line can be obtained by the line parameters and related measuring equipment. The electrical distance $l$ has many complicated branch lines due to the structure of the distribution network. There is no direct and convenient measurement method. Therefore, a Floyd algorithm based method for calculating the shortest electrical distance from the live working point of the distribution network to the substation is proposed.

The method process is as follows:

(1)The branch nodes of the line are numbered $(1,2, \cdots, n)$ with the substation as the center, the total number of nodes is $\mathrm{n}$, and the node set is $\mathrm{N}$;

(2)The distance matrix D is obtained by the adjacent distance between the nodes;

(3)Define the shortest path matrix as $D_{\min }$, let $D_{\text {min }}=D$;

(4)Determine whether (1) is true when seeking the shortest path;

$$
\left\{\begin{aligned}
D_{\text {min }}(i, k) & \geq D_{\text {min }}(i, j)+D_{\text {min }}(j, k) \\
j & \in N, i \neq j, j \neq k
\end{aligned}\right.
$$

(5)If equation (1) holds, then equation (2) satisfies the condition, otherwise equation (3) satisfies the condition.;

$$
\begin{aligned}
& \left\{\begin{array}{c}
D_{\text {min }}(i, k)=D_{\text {min }}(i, j)+D_{\text {min }}(j, k) \\
j \in N, i \neq j, j \neq k
\end{array}\right. \\
& D_{\min }(i, k)=D_{\min }(i, k)
\end{aligned}
$$

Let $j=1,2, \ldots, n, i \neq j, j \neq k$. Search all nodes in turn. If the elements in matrix $D_{\text {min }}$ have not changed during the search, $D_{\min }$ is the final shortest path matrix. If an element changes, jump to step (4) until all elements are no longer Until there is a change;

(6) Output matrix $D_{\text {min }}$. The arbitrary element of the matrix is the shortest distance from branch node $\mathrm{i}$ to branch node $\mathrm{j}$.

$$
\left\{\begin{array}{c}
D_{\min }(i, j) \\
i \in N \\
j \in N
\end{array}\right.
$$

By inputting the value through the remote humanmachine interchange module, the central computing module can calculate the electrical distance from the working point to the outlet end of the substation, and then calculate the exact value of the input current. The current injection module can inject the current into the neutral point of the substation. Realize zero point of the operating point.

$$
\operatorname{dis}=D_{\min }\left(1, w_{u}\right)+d_{u}
$$

After obtaining the distance between the branch nodes of the line through the pre-project construction data and the post-measurement calibration, the shortest electrical distance from any branch node to the exit end of the line can be conveniently and quickly obtained, and input into the active inverter device can realize precise voltage regulation and operation. The point potential is zero.

\section{The zero-potential non-blackout operation method for distribution network}

To regulate the voltage of any working phase, a power supply device or a power electronic device can be used to inject current $\left(\dot{I}_{i}\right)$ into the neutral point. Taking the $\mathrm{C}$ phase as an example, the vector diagram is used for analysis. The current is injected into the neutral point through the grounding transformer, and the zerosequence voltage will appear in each phase. It will cause the neutral point to ground voltage $\left(\dot{U}_{\mathrm{N}}\right)$ to shift. The power supply and load are not affected by the phasedown of the C-phase. Therefore, the non-effective grounding distribution system has the natural advantage of maintaining the line voltage symmetric operation when single-phase step-down, and the single-phase stepdown symmetrical operation is feasible.

In Fig. 1, the C-phase line needs to be energized. Assume that the operating point voltage is $\dot{U}_{\mathrm{f}}$, the neutral point voltage is $\dot{U}_{\mathrm{N}}$, the line unit impedance is z, the voltage drop between the power bus and the operating point is $\Delta \dot{U}$, and the three-phase power supply voltage is respectively $\dot{E}_{A} 、 \dot{E}_{B} 、 \dot{E}_{C}$.

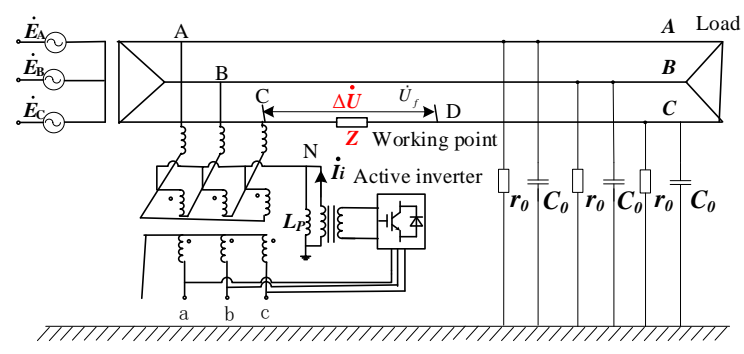

Figure 1. Working point zero potential control schematic

For distribution lines of $35 \mathrm{kV}$ and below, the impedance between the substation and the operating 
point in Figure 1 can be obtained by multiplying the unit impedance $\mathrm{Z}$ by the electrical distance 1 . When the working phase current is $\dot{I}_{c}, \Delta \dot{U}$ can be obtained by Ohm's law:

$$
\Delta \dot{U}=\dot{I}_{C} Z l
$$

The KVL theorem for c-phase circuits is available:

$$
\dot{U}_{N}=\dot{U}_{d}-\dot{E}_{C}+\Delta \dot{U}
$$

The neutral point can be obtained by using the KCL theorem:

$$
\dot{I}_{i}=\left(\dot{E}_{A}+\dot{U}_{N}\right) Y_{0}+\left(\dot{E}_{B}+\dot{U}_{N}\right) Y_{0}+\left(\dot{E}_{C}+\dot{U}_{N}-\Delta \dot{U}\right) Y_{0}+\dot{U}_{N} Y_{L}
$$

Where, $\dot{I}_{i}$ is the current injected into the neutral point by the active inverter, $Y_{0}$ is earth admittance when the power grid is in normal operation, $Y_{L}$ is ground admittance of arc suppression coil.

$\dot{U}_{N}$ can be expressed as:

$$
\dot{U}_{N}=\frac{\dot{I}_{i}+\Delta \dot{U} Y_{0}}{3 Y_{0}+Y_{L}}
$$

It can be known from equation (9) that the neutral point voltage $\dot{U}_{N}$ can be regulated when zero sequence current is injected into the neutral point. When $\dot{U}_{N}=-\dot{E}_{C}+\Delta \dot{U}$, according to equation (7), the voltage at the operation point at this time is 0 , that is, when the change of zero sequence voltage is $\dot{U}_{d}$, the potential at the corresponding operation point is zero. Therefore, if and only if when the zero-sequence current is injected, the amount of change in the zero-sequence voltage is adjusted to the opposite of the sum of the operating phase power supply voltage and the operating phase line voltage drop, the operating point voltage is guaranteed to be zero, creating for subsequent manual safety operations. condition.

\section{Verification of simulation}

In PSCAD, a schematic diagram of the zero-potential charging operation of the $10 \mathrm{kV}$ distribution network shown in Figure 1 is built. The neutral point is taken from the YnY11 grounding transformer. The power supply device is connected between the neutral point and the ground, and is respectively output by a voltage source and a current source. Set the total length of the line to $15 \mathrm{~km}$, carry out live operation at point $\mathrm{C}$ of phase $\mathrm{C}$, and point $\mathrm{D}$ is $3 \mathrm{~km}$ away from the line exit.

Under the same injection mode, the calculation error of the distance between the substation and the working point is assumed to be $20 \mathrm{~m}, 110 \mathrm{~m}, 205 \mathrm{~m}$. After the regulation, the grounding line is set at the working point to observe the change of the operating point voltage during the whole operation. Set the simulation parameters as shown in Table 1.

Table 1. Simulation parameter table

\begin{tabular}{cc}
\hline Variable name & Numerical value \\
\hline Single relative leakage resistance $r_{0}$ & $4400 \Omega$
\end{tabular}

\begin{tabular}{c|c} 
Single relative capacitance $C_{0}$ & $14.9 \mu \mathrm{F}$ \\
Line unit length resistance & $r_{1}$ \\
Line unit length reactance $x_{1}$ & $0.0525 \Omega / \mathrm{km}$ \\
Arc suppression coil inductance Lp & $0.415 \Omega / \mathrm{km}$ \\
\hline
\end{tabular}

\subsection{The calculation error is $20 \mathrm{~m}$}

Assume that when calculating the distance between the substation and the working point, the calculation error is $20 \mathrm{~m}$, that is, the actual control point is $3.02 \mathrm{~km}$ away from the line exit. The voltage source is used to realize the zero-potential charging operation, and the simulation parameter is substituted into the formula (5) to calculate the voltage source size as $\dot{U}_{i}=6.10 \angle-60.05^{\circ} \mathrm{kV}$. The simulation duration is set to $1.2 \mathrm{~s}$. At $0.4 \mathrm{~s}$, the voltage source is used to regulate the operating point voltage. At $0.8 \mathrm{~s}$, the grounding line is set on the ground near the working point. The voltage changes of the non-working phase (A and $\mathrm{B}$ phases) are shown in Figure 2 and Figure 3. The operating point $(\mathrm{C}$ phases) voltage change is shown in Figure 4.

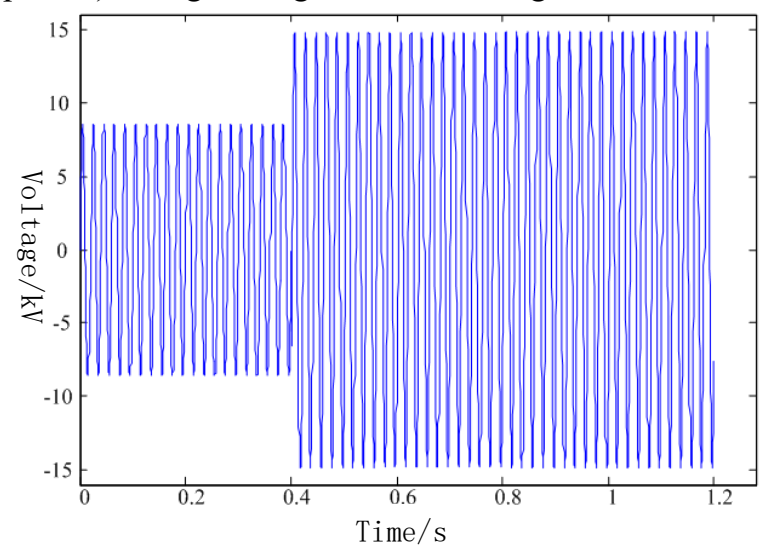

Figure 2. The voltage change of phase A

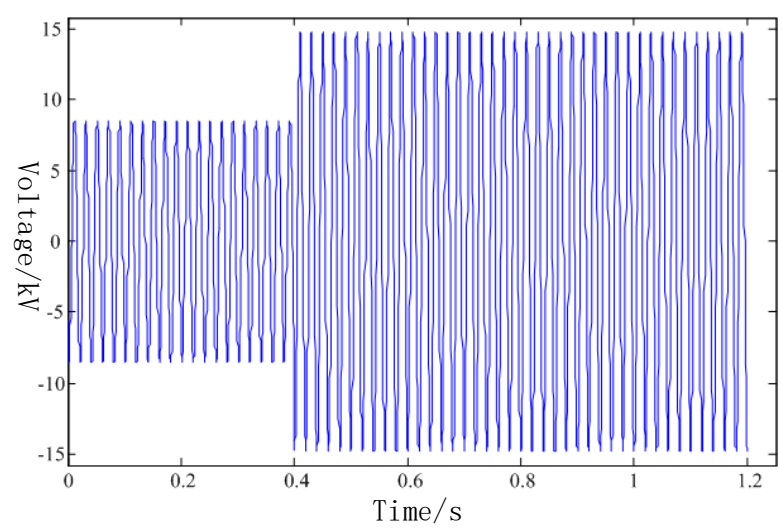

Figure 3. The voltage change of phase B

It can be seen from Fig. 2 and Fig. 3 that the nonworking phase voltage is normal before $0.4 \mathrm{~s}$. After $0.4 \mathrm{~s}$, the voltage source starts to inject the current regulation voltage, and the $\mathrm{A}$ and $\mathrm{B}$ phase voltages are higher than 
the phase voltage under normal operation, close to the line voltage. At the $8 \mathrm{~s}$ time, the grounding line is set on the analog working point, which is equivalent to a single-phase grounding fault. In this case, the nonworking phase voltage rises to the line voltage.

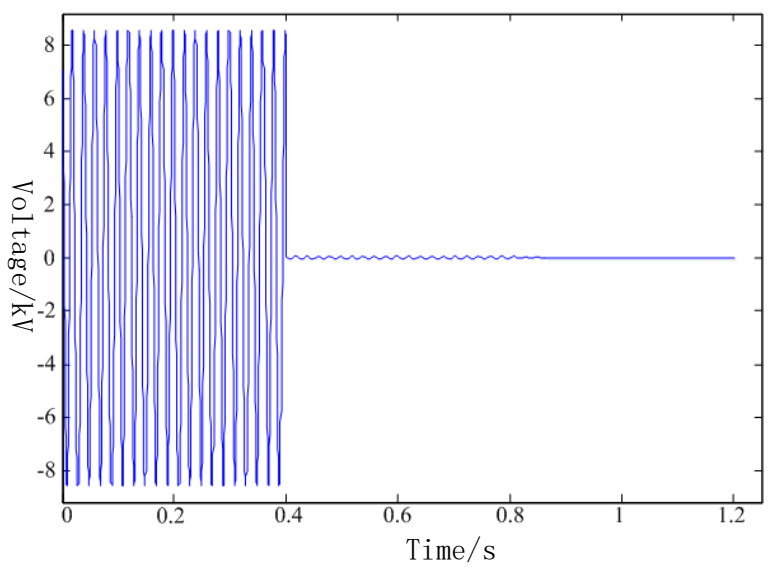

Figure 4. Operating point voltage change

It can be seen from Fig. 4 that before $0.4 \mathrm{~s}$, the effective value of the operating point voltage is $6.05 \mathrm{kV}$, and the voltage source is used to inject current at $0.4 \mathrm{~s}$. Since the actual regulation point is $3.02 \mathrm{~km}$ away from the line exit, the actual operating point $\mathrm{D}$ voltage is not zero. Potential, at this point, the effective value of the point $\mathrm{D}$ of the operating point is $40.13 \mathrm{~V}$. At $0.8 \mathrm{~s}$, the grounding line is set on the analog working point. It can be seen that after setting the grounding wire, the voltage at point $\mathrm{D}$ is around $0 \mathrm{~V}$.

\subsection{The calculation error is $110 \mathrm{~m}$}

Assume that when calculating the distance between the substation and the working point, the calculation error is $110 \mathrm{~m}$, that is, the actual control point is $3.11 \mathrm{~km}$ away from the line exit. The voltage source is used to realize the zero-potential charging operation, and the simulation parameter is substituted into the formula (5) to calculate the voltage source size as $\dot{U}_{i}=6.11 \angle-60.06^{\circ} \mathrm{kV}$.The simulation duration is set to $1.2 \mathrm{~s}$. At $0.4 \mathrm{~s}$, the voltage source is used to regulate the operating point voltage. At $0.8 \mathrm{~s}$, the grounding line is set on the ground near the working point. The voltage changes of the non-working phase (A and B phases) are shown in Figure 5 and Figure 6 . The operating point $(\mathrm{C}$ phases) voltage change is shown in Figure 7.

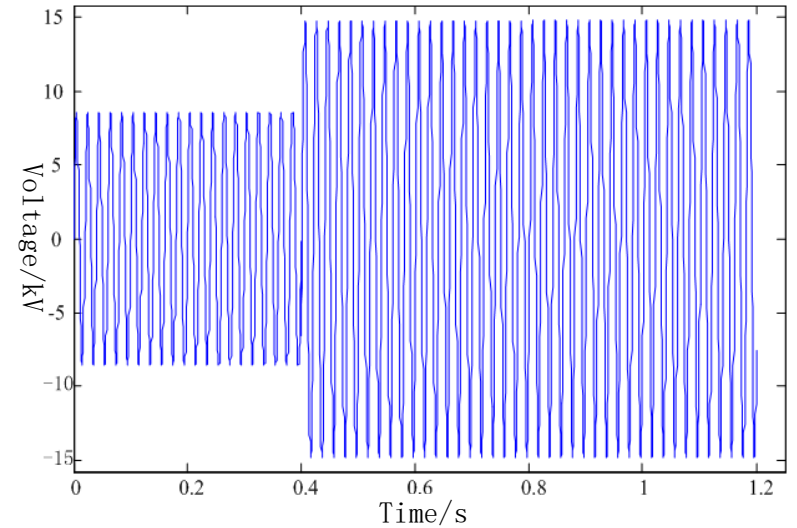

Figure 5. The voltage change of phase A

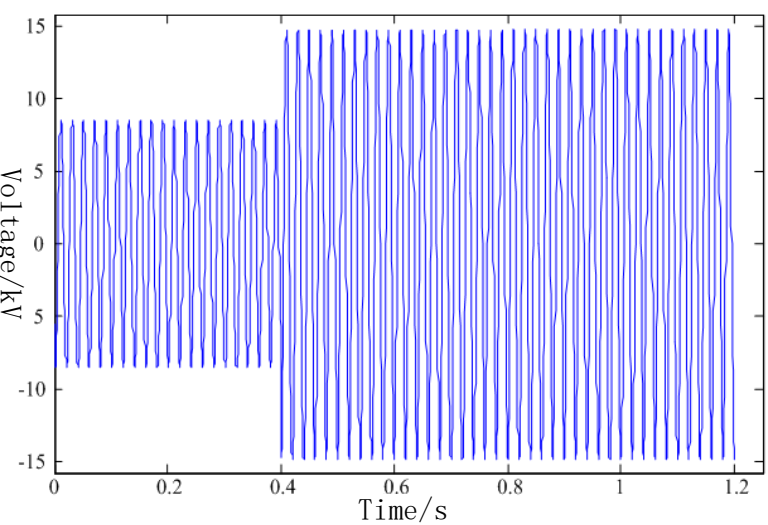

Figure 6. The voltage change of phase B

It can be seen from Fig. 5 and Fig. 6 that the nonworking phase voltage is normal before $0.4 \mathrm{~s}$. After $0.4 \mathrm{~s}$, the voltage source starts to inject the current regulation voltage, and the A and B phase voltages are higher than the phase voltage under normal operation, close to the line voltage. At the $8 \mathrm{~s}$ time, the grounding line is set on the analog working point, which is equivalent to a single-phase grounding fault. In this case, the nonworking phase voltage rises to the line voltage.

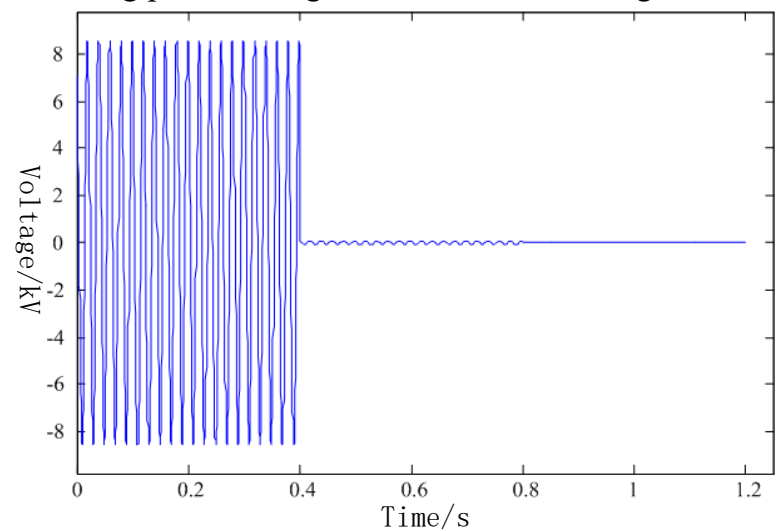

Figure 7. Operating point voltage change

It can be seen from Fig. 7 that before $0.4 \mathrm{~s}$, the effective value of the operating point voltage is $6.05 \mathrm{kV}$, and the voltage source is used to inject current at $0.4 \mathrm{~s}$. 
Since the actual regulation point is $3.11 \mathrm{~km}$ away from the line exit, the actual operating point $\mathrm{D}$ voltage is not zero. Potential, at this point, the effective value of the point $\mathrm{D}$ of the operating point is $195.48 \mathrm{~V}$. At $0.8 \mathrm{~s}$, the grounding line is set on the analog working point. It can be seen that after setting the grounding wire, the voltage at point $\mathrm{D}$ is around $0 \mathrm{~V}$.

\subsection{The calculation error is $205 \mathrm{~m}$}

Assume that when calculating the distance between the substation and the working point, the calculation error is $205 \mathrm{~m}$, that is, the actual control point is $3.205 \mathrm{~km}$ away from the line exit. The voltage source is used to realize the zero-potential charging operation, and the simulation parameter is substituted into the formula (5) to calculate the voltage source size as $\dot{U}_{i}=6.10 \angle-60.07^{\circ} \mathrm{kV}$. The simulation duration is set to $1.2 \mathrm{~s}$. At $0.4 \mathrm{~s}$, the voltage source is used to regulate the operating point voltage. At $0.8 \mathrm{~s}$, the grounding line is set on the ground near the working point. The voltage changes of the non-working phase (A and $\mathrm{B}$ phases) are shown in Figure 8 and Figure 9. The operating point $(\mathrm{C}$ phases) voltage change is shown in Figure 10.

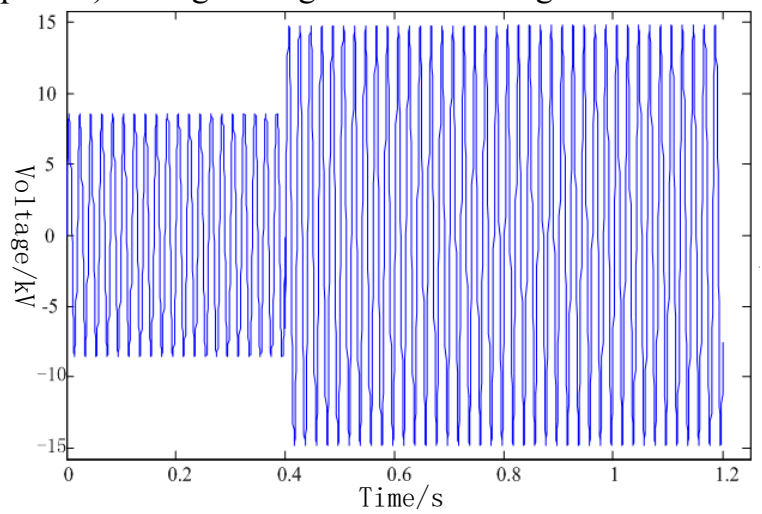

Figure 8. The voltage change of phase A

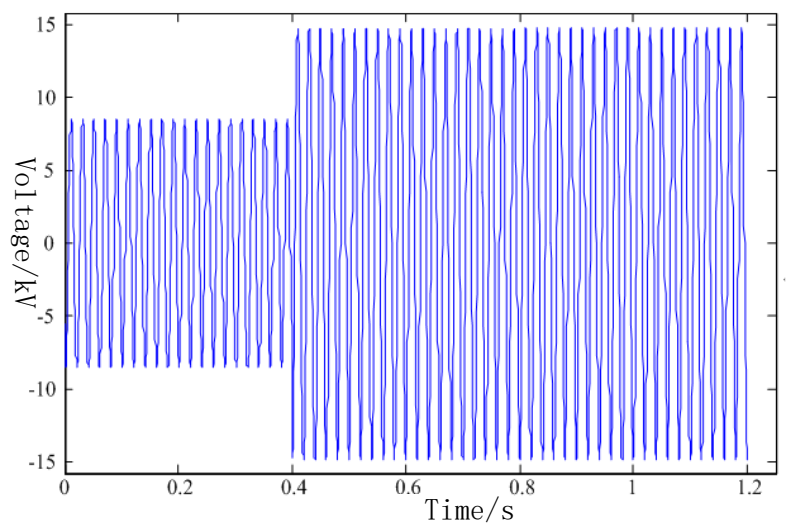

Figure 9. The voltage change of phase B

It can be seen from Fig. 8 and Fig. 9 that the nonworking phase voltage is normal before $0.4 \mathrm{~s}$. After $0.4 \mathrm{~s}$, the voltage source starts to inject the current regulation voltage, and the $\mathrm{A}$ and $\mathrm{B}$ phase voltages are higher than the phase voltage under normal operation, close to the line voltage. At the $8 \mathrm{~s}$ time, the grounding line is set on the analog working point, which is equivalent to a single-phase grounding fault. In this case, the nonworking phase voltage rises to the line voltage.

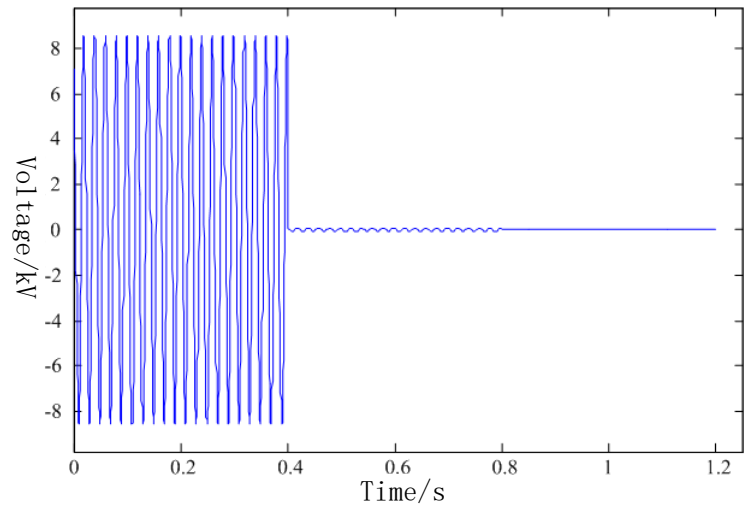

Figure 10. Operating point voltage change

It can be seen from Fig. 10 that before $0.4 \mathrm{~s}$, the effective value of the operating point voltage is $6.05 \mathrm{kV}$, and the voltage source is used to inject current at $0.4 \mathrm{~s}$. Since the actual regulation point is $3.205 \mathrm{~km}$ away from the line exit, the actual operating point $\mathrm{D}$ voltage is not zero. Potential, at this point, the effective value of the point $\mathrm{D}$ of the operating point is $378.75 \mathrm{~V}$. At $0.8 \mathrm{~s}$, the grounding line is set on the analog working point. It can be seen that after setting the grounding wire, the voltage at point $\mathrm{D}$ is around $0 \mathrm{~V}$.

\section{Conclusion}

(1)The injection of the calculated injection current through the voltage source can achieve precise control of the operating point voltage;

(2)When calculating the distance between the substation and the working point, if a small error occurs (the error distance is less than $20 \mathrm{~m}$ ), the operating point voltage can still be guaranteed below the safe voltage;

(3)If the grounding wire is set on the ground during the live working, the working point will be zero potential regardless of the distance error between the substation and the working point. Therefore, in order to ensure the safety of the operator during live working, the grounding wire should be set locally.

\section{References}

1. Poorinima S, Pushpalatha D, Power system transient stability improvement of two machine system using fuzzy logic controlled statcom[C], International Journal of Electrical and Electronic Engineering \& Telecommunications, 2014, 3(2): 1-11.

2. Chayanika Baruah, Dipankar Chanda, A comparative study of wavelet transform techinque and svd in the estimation of power system harmonics and interharmonics[C], International Journal of Electrical and Electronic Engineering \& Telecommunications, 2014, 3(4): 22-29. 
3. Nasibov F, Yorukoglu S, Gul O, et al. A feasibility study of live working in Turkish electricity distribution system[C]// International Conference on Live Maintenance. 2017.

4. Fang $\mathrm{Y}$, Wang $\mathrm{L}$, Li R, et al. AC Flashover Performance of FRP Hot Stick for Live Working in High Altitude Areas[J]. IEEE Access, 2019, PP(99):1-1.

5. Dai X, Li X, Li Y, et al. Impedance matching range extension method for maximum power transfer tracking in IPT system[C]// Power Electronics Conference. IEEE, 2017.

6. Kon S, Yamada T. Expansion of the Impedance and Frequency Measurement Ranges of AC Shunt Resistors[J]. IEEE Transactions on Instrumentation and Measurement, 2017, 66(6):1254-1259.

7. Liao Y, Kezunovic M. Optimal Estimate of Transmission Line Fault Location Considering
Measurement Errors[J]. IEEE Transactions on Power Delivery, 2007, 22(3):1335-1341.

8. Teng J H, Huang W H, Luan S W. Automatic and Fast Faulted Line-Section Location Method for Distribution Systems Based on Fault Indicators[J]. IEEE Transactions on Power Systems, 2014, 29(4):1653-1662.

9. Zhang Y, Zhang Q, Song W, et al. Transmission line fault location for double phase-to-earth fault on nondirect-ground neutral system[J]. IEEE Transactions on Power Delivery, 2000, 15(2):520-524.

10. Pramudya F S, Pan J, Devlin A T. Estimation of Significant Wave Height of Near-Range Traveling Ocean Waves Using Sentinel-1 SAR Images[J]. IEEE Journal of Selected Topics in Applied Earth Observations and Remote Sensing, 2019, 12(4):1067-1075. 